\title{
Addressing health and social care during and beyond COVID-19: the importance of implementation science
}

\section{Helen Skouteriss,b,c}

a Health and Social Care Unit, School of Public Health and Preventive Medicine, Monash University, Melbourne, VIC, Australia

b Warwick Business School, University of Warwick, Coventry, UK

c Corresponding author: helen.skouteris@monash.edu

\section{Article history}

Publication date: March 2021

Citation: Skouteris H. Addressing health and social care during and beyond COVID-19: the importance of implementation science. Public Health Res Pract. 2021;31(1):e3112103. https://doi. org/10.17061/phrp3112103

\section{Key points}

- Implementation science plays a pivotal role in the prevention and treatment of, and recovery from, COVID-19, especially in safeguarding the most vulnerable in our community

- To enact effective evidence-based interventions and communication strategies, there is a need to understand not only what but also how to meaningfully implement multifaceted solutions at pace and scale

- We need a strong participatory and codesigned response with representatives from all population groups (including those who are oppressed, marginalised and vulnerable) when implementing interventions to drive effective behaviour change to enable recovery from the pandemic

\section{Abstract}

Implementation science is increasingly relevant and important as we move beyond the immediate response to, and curtailing of, the coronavirus disease 2019 (COVID-19) pandemic. Understanding how to prevent the spread of the virus, and treating and improving the lives of those infected, requires translating knowledge into action, and implementing evidence-based interventions. We know the direct impacts of COVID-19, and associated interventions to address it, will not be felt equally across all population groups. Hence, it is proposed that the integration of health and social sciences is fundamental to mitigate potential adverse impacts of interventions for COVID-19. In this paper I argue that the involvement of consumers and the community in the quest to combat and recover from the COVID-19 pandemic will enable social equity, as demonstrated by a case study.

\section{Background}

The relevance and importance of implementation science in the global response to the coronavirus disease 2019 (COVID-19) pandemic is clear, given the robust evidence that is now starting to emerge. ${ }^{1}$ Implementation science focuses on translating research into practice, understanding how interventions are implemented, and what influences implementation outcomes. ${ }^{2}$ Understanding how to prevent the spread of the virus and treating and improving the lives of those infected requires us to translate knowledge into action and to implement evidence-based interventions. Wensing et al. ${ }^{1}$ identified a number of areas that will likely be crucial for implementation science as we move beyond the immediate response to and curtailing of COVID-19. One of these areas is mitigating the adverse impacts of interventions for COVID-19, such as inequalities in access to healthcare, or inequitable treatment for vulnerable populations. Here, I highlight the importance of implementation science in integrating health and social care, and provide a specific case study to demonstrate how this approach can 
improve equity in health outcomes for some of our most vulnerable young people.

\section{A roadmap to recovery: applying an equity lens to the Australian COVID-19 response}

In April 2020, I was invited to work with more than 100 scholars from Australia's Group of Eight leading universities to inform the many decisions our Federal, State and Territory Governments need to make to manage and recover from the COVID-19 pandemic in Australia. The Roadmap to recovery: a report for the nation was developed. ${ }^{3}$ We know that the impacts of COVID-19 will not be felt equally across all population groups. In this report, we identified a number of key groups that require special considerations and support in the recovery phase of the response to COVID-19, including women who are pregnant, women at risk of domestic violence, children and young people - specifically those living in out-of-home care - older adults, and those living in residential aged care. Eleven recommendations for targeted policy strategies and support were made based on evidence from key findings that confirm the need for action to safeguard our most vulnerable populations at a time of heightened health risk. In addition, it was made clear that success of recovery will depend on the extent to which the public partner with governments to adapt their behaviours until a vaccine is widely available enabling restrictions to be relaxed. The need to focus on how public health messages are communicated effectively, across all societal groups, and how targeted policy strategies and support for our key populations are developed and scaled, speaks to the importance of implementation science.

\section{Applying implementation science to reduce inequalities in the COVID-19 response}

Dissemination of information that is translated into different languages is important in the communication of pandemic preventive strategies, however information alone will not lead to behaviour change. Effective mass public health communication requires an understanding of behavioural psychology principles as well as information about how to tailor key messages to the various populations within a society; there is no one-size-fits-all approach for diverse populations. ${ }^{4}$ Implementation science is needed to convert the recommendations for equity of access and outcomes in health and psychosocial support outlined in the Roadmap to recovery report ${ }^{3}$ into action. That is, to drive evidence-based health and social care interventions and communication strategies into practice, there is a need to understand not only what but also how to meaningfully implement multifaceted solutions at pace and scale to mitigate the heightened risk of long-term adverse effects of COVID-19 on key populations. Embarking on the journey to uncover the how ineludibly takes us into multilevel, multisectoral and complex territory. ${ }^{4}$ This is especially evident when there's a need to embed traumaresponsive health-focused interventions that reach, and are well received by, our most vulnerable populations across and within social welfare and community service settings. ${ }^{5}$

Implementation science frameworks are predominantly focused on addressing the following aims²:

1) To inform and guide the implementation of an intervention by specifying the phases and stages needed for translating research into practice (process theories);

2) To reveal what influences the implementation outcomes (determinant theories); and

3) To assess how interventions are implemented, that is, to assess the process of implementation (evaluation theories).

However, what is often missing from these frameworks is the end-user relevance ${ }^{2}$; this is critically important to combat COVID-19, since outbreaks of the disease in Australia, as in other countries globally, have been characterised by over-representation of vulnerable populations. $^{3}$

Stakeholder engagement and genuine partnership - including the voice of those with a lived experience need to be embedded throughout the implementation process so that new knowledge is implemented proactively, translated and scaled into changes in practice and policy. By situating consultation, collaboration, and codesign across the implementation process, research, translation, clinical, and public health activities are more likely to be responsive to, and relevant for, stakeholder and end-user needs, and therefore are more likely to deliver impact. ${ }^{6}$ That is why the Roadmap to recovery report ${ }^{3}$ recommended a strong participatory and codesigned response. In relation to COVID-19, this is particularly important for behaviours such as getting tested if symptoms develop, physical distancing, sanitising and wearing masks. Partnerships between health authorities/agencies, government stakeholders, researchers and target populations can help to develop an understanding of what drives these behaviours, in order to engage diverse community members in effective behaviour change. $^{7}$

Stakeholder engagement and codesign are principles and concepts that are well understood and adopted by governments globally, however moving evidencebased public health knowledge tools and interventions/ programs into practice is challenging. The challenges arise for many reasons, including: complex pathways of action and intervention in public health; political influence and commitment; budgetary restraints and 
commitment; workforce capacity (to understand research and the science of implementation); a lack of integration and partnerships between public- and private-sector organisations and across health and social care; generalisation and applicability to local populations; and timely and accurate communication of information to all key stakeholders to mobilise engagement across populations and effect behaviour change. ${ }^{8}$

\section{Reducing disparities for}

\section{vulnerable youth - implementation science in action}

Children and young people, specifically those living in out-of-home care $(\mathrm{OOHC})$ were identified as a key population in the Roadmap to recovery report. ${ }^{3}$ This population experiences higher rates of adverse physical and mental health outcomes, and once they leave care, they continue to experience disadvantage with respect to educational achievements, training and employment, housing, and health and wellbeing, compared with typically developing young people. ${ }^{9}$ COVID-19 and the interventions addressing the pandemic are likely to disproportionately impact this population - however, integrating health and social sciences in the provision of support can mitigate potential adverse effects.

The Healthy Eating Active Living (HEALing) Matters program illustrates an approach where consumers and the community are actively involved throughout the implementation journey of a project (Box 1). HEALing Matters provides information and practical resources to help young people living in $\mathrm{OOHC}$ make positive choices and behaviour changes in relation to their health and wellbeing. The intervention was designed in recognition that the implementation approach to bring about change must match the challenges that need to be overcome to achieve change. ${ }^{10}$ Therefore, HEALing Matters was codesigned, every step of the way with key stakeholders across all levels of the health and social care settings. This helped to identify the challenges and needs that had to be addressed to bring about change in the lifestyle behaviours of young people living in $\mathrm{OOHC}$. The case study demonstrates that moving from a behavioural to a sociological approach is crucial in an intervention to address the risk of overweight and obesity in children living in $\mathrm{OOHC}$. Similarly, health and social care should be integrated into the implementation strategy for COVID-19 interventions to appropriately address the unique needs of our vulnerable populations and ensure equity in health outcomes.

Given there is emerging evidence that obesity-related conditions appear to worsen the effects of COVID-19 ${ }^{14}$, HEALing Matters has become even more strategically pertinent in the bid to improve the lives of those in care and prevent long-term chronic diseases and associated morbidities.
Box 1. A case study: implementation and scale-up of an intervention for vulnerable young people

Overweight and obesity is a health risk experienced by young people who are living in $\mathrm{OOHC}$ that has previously been overlooked. In 2014, research in Victoria showed children living in $\mathrm{OOHC}$ are at substantially increased risk of excessive weight gain and associated morbidity; almost two-thirds (62.8\%) of the young people living in $\mathrm{OOHC}$ were overweight/obese compared with $28.9 \%$ of the national adolescent population. ${ }^{11}$ In response, we (specifially Professor Skouteris and Dr Rachael Green) codesigned and now lead the implementation and scale-up of the online Healthy Eating Active Living (HEALing) Matters program. ${ }^{12}$

A behavioural health response alone to obesity prevention for young people in $\mathrm{OOHC}$ did not bring about significant change in their lifestyle behaviours, as it did not acknowledge the broader context of the unqiue social challenges and disadvantage that they face. Therefore, we quickly realised that partnering with the community and consumers, involving the voice of lived experience, and moving away from a behavioural approach to a sociological approach to inform the intervention was needed. HEALing Matters now recognises that food and physical activity can be powerful ways of demonstrating trust, value, predictability, a sense of belonging, and the provision of support and care that is attuned to the needs of the young people. ${ }^{13} \mathrm{HEALing}$ Matters is now delivered within a framework informed by attachment, trauma and resilience theories. ${ }^{5}$

We have also moved away from a focus on implementation in relation only to individual behaviour change among the young people and their carers to more effectively address the broader challenges and barriers that young people experience in the $\mathrm{OOHC}$ context. Instead, we have embedded an understanding of the success of the implementation strategy in the adoption and uptake of HEALing Matters at the following levels: 1) system and/or divisional government; 2) organisational; 3) residential home; and 4) individual. This design is pertinent to sustainability and scale-up considerations that will help us to answer questions of what works, for whom, why, and in what circumstances. ${ }^{13}$

\section{Conclusion}

The integration of health and social sciences for health and social care improvement is fundamental to mitigate adverse impacts of interventions for COVID-19. Indeed, for our vulnerable populations, social justice is central to public health and crucial for effective COVID-19 prevention, testing and treatment. ${ }^{15}$ This is highlighted in a case study, which found that a behavioural health response alone to obesity prevention for young people in $\mathrm{OOHC}$ does not work. ${ }^{5}$ The integration of health and social care throughout the implementation journey including the involvement of consumers and community 
- will enable the quest to combat and recover from the COVID-19 pandemic to perpetuate equity of access and outcomes in health and psychosocial support.

\section{Peer review and provenance}

Externally peer reviewed, not commissioned.

\section{Competing interests}

None declared.

\section{Author contributions}

HS is the sole author of the paper.

\section{References}

1. Wensing M, Sales A, Armstrong R, Wilson P. Implementation science in times of COVID-19. Implement Sci. 2020;15(1):42.

2. Nilsen P. Making sense of implementation theories, models and frameworks. Implement Sci. 2015;10(1):53.

3. Group of Eight Australia. COVID-19 roadmap to recovery. a report for the Nation. Canberra: Go8; 2020 [cited 2020 Jul 19]. Available from: go8.edu.au/wp-content/ uploads/2020/05/Go8-Road-to-Recovery.pdf

4. Chambers DA. Considering the intersection between implementation science and Covid-19. Implement Sci. 2020;1:1-4.

5. Pizzirani B, Green (nee Cox) R, O'Donnell R, Skouteris H. Healthy lifestyle programs in out-of-home care: implementing preventative trauma-informed approaches at scale. Aust Soc Work. 2020:Online early.

6. Lim S, Morris H, Pizzirani B, Kajewski D, Lee WK, Skouteris $\mathrm{H}$. Evaluating hospital tools and services that were co-produced with patients: a rapid review. Int J Qual Health Care. 2020;32(4):231-9.
7. Wild A, Kunstler B, Goodwin D, Skouteris H. We ask multicultural communities how to best communicate COVID-19 advice. Here's what they told us. Melbourne: The Conversation; 2020 [cited 2020 Aug 11]. Available from: theconversation.com/we-asked-multiculturalcommunities-how-best-to-communicate-covid-19-adviceheres-what-they-told-us-142719

8. Atkins L, Kelly MP, Littlford C, Leng G, Michie S. Reversing the pipeline? Implementing public health evidence-based guidance in English local government. Implement Sci. 2017;12(1):63

9. Smales M, Savaglio M, Morris H, Bruce L, Skouteris H, Green (nee Cox) R. "Surviving not thriving": experiences of health among young people with a lived experience in out-of-home care. Int J Adolesc Youth. 2020;25(1):809-23.

10. Wensing M, Grol R. Knowledge translation in health: how implementation science could contribute more. BMC Med. 2019;17(1):88.

11. Cox R, Skouteris H, McCabe M, Fuller-Tyszkiewicz M, Jones AD, Hardy LL. Rates of overweight and obesity in a sample of Australian young people and their carers in out-of-home residential care. Aus NZ J Publ Health. 2014;38(6):591-2.

12. Monash University. Healing Matters. Melbourne: Monash University; 2020 [cited 2020 Aug 11]. Available from: healing-matters.org

13. Pizzirani B, O'Donnell R, Bruce L, Breman R, Smales M, Xie J, et al. The large-scale implementation and evaluation of a healthy lifestyle program in residential out-of-home care: study protocol. Int J Adolesc Youth. 2020;25(1):396406.

14. Stone JR. Social justice, triage, and Covid-19: ignore lifeyears saved. Med Care. 2020;58(7):579-81.

15. Caussy C, Pattou F, Wallet F, Simon C, Chalopin S, Telliam C. Prevalence of obesity among adult inpatients with COVID-19 in France. Lancet Diabetes Endocrinol. 2020;8(7):562-4.

\section{Copyright: (c) $(\mathbf{0})(0)$}

(C) 2021 Skouteris. This article is licensed under the Creative Commons Attribution-NonCommercial-ShareAlike 4.0 International Licence, which allows others to redistribute, adapt and share this work non-commercially provided they attribute the work and any adapted version of it is distributed under the same Creative Commons licence terms. See: www.creativecommons.org/licenses/by-nc-sa/4.0/ 